\title{
AN OFFLINE INSPECTION AND DISPOSITION MODEL INCORPORATING DISCRETE WEIBULL DISTRIBUTION AND MANUFACTURING VARIATION
}

\author{
Chih-Hsiung Wang Chen-Chien Hung \\ National Pingtung Institute of Commerce
}

(Received December 27, 2006; Revised October 11, 2007)

\begin{abstract}
To solve the quality control problem for a batch produced from an unreliable production system, an economic offline inspection and disposition (ID) model was previously proposed where the process is assumed to have a constant failure rate, i.e., a geometric shift distribution. Through setting the proper value of parameters in the ID model, three commonly used policies: cost minimizing, perfect information and zero-defects are readily obtained. To facilitate the adoption of the developed ID model in practical applications, this study investigated the ID model in which two additional features are considered: (i) the process has a non-constant failure rate (NCFR). (ii) A manufacturing variation (MV) in the process exists. Two sets of recursive cost equations are obtained to establish an algorithm for the optimal ID policy of a given production lot. Numerical examples are used to show that MV and/or NCFR have significant effects on the minimum cost. The results also show that it is infeasible to reach the perfect information policy once MV exists, but that the zero-defects policy remains feasible. Finally, a concluding remark is made.
\end{abstract}

Keywords: Quality control, optimization

\section{Introduction}

When an unreliable production process is considered, it may shift from an in-control to an out-of-control state. This will result in the production of a higher proportion of defective units. When no inspection policy was considered, Porteus [5] used a small lot to control the number of defective units. Based on Porteus's [5] model, the defective unit costs were considered under a free repair warranty policy and an inspection/repair policy by Djamaludin et al. [2] and Wang and Sheu [8], respectively. To achieve a balance between the number of the defective units (i.e., defective cost) and the quality related control cost (i.e., inspection and disposition), the use of an off-line inspection (or testing) policy is sometimes inevitable for certain industrial production situations, e.g., food (e.g., see Wang et al. [10] and Brosnan and Sun [1]), or electronic products, which need to burn in their quality. In general, an off-line policy is used when it takes a long period of time to identify product quality, and this makes an on-line process control unfeasible.

Without considering manufacturing variation, many studies (e.g., see He et al. [3]) have attempted to solve the off-line inspection problem by finding the process shift point. All units produced after the shift point are defective and should be rejected and vise versa. This is referred to as the perfect information policy. Unlike these previous works, Raz et al. [6] focused on the balance between the inspection cost and disposition cost. Their policy is to continue the following ID actions until all units have been dispositioned (i.e., accepted or rejected). Under the objective of cost minimization, a unit is selected from the remaining batch to be inspected. All units prior the inspected unit are accepted (or remained) and all units after it remain (or are rejected) if the unit has been inspected to 
be conforming (or non-conforming). Of course, whether to inspect a unit or not in a batch is based on comparison of the resulting cost to that of the no inspection policy. Under the no inspection policy, the break-even point (BEP) in the batch is first calculated, where the $\mathrm{BEP}$ is the point when the penalty of incorrect acceptance and incorrect rejection are equal. Second, according to the deteriorating property of the process, all the units after the BEP are rejected and others accepted. Raz et al. [6] took advantage of the memoryless property when the process has a geometric shift distribution. They developed two sets of recursive equations which do not need to record the original position of each unit in the batch for the optimal ID policy. Through setting proper values on the unit penalty cost of incorrect acceptance and rejection in Raz et al.'s [6] model, the following three policies are obtained: cost minimizing, perfect information and zero-defects, where the zero-defects policy is that all accepted units are known to be conforming.

However, when product inspection cannot perfectly reflect the process status while producing the inspected item, Raz et al.'s [6] inspection/disposition (RID) model would be not be pertinent. This implies that once the possibility of inspection errors (IEs) or manufacturing variation (MV) exists, the RID model should be modified before it is used. IEs or MV are present in different types of production environments. For example, IEs usually arise during food products inspection (e.g., see [1]); while MV exists as a part of the nature of the manufacturing semiconductors.

Recently, Sheu et al.[7] and Wang [9] introduced two types of IEs into the RID model to see what are the effects of IEs on optimal inspection policy, where the process shift distribution is assumed to be geometric. This is not the same as this study, where we focus on a situation where the process has MV and possesses a non-constant failure rate. This is motivated by the following deficiencies of the RID model: (i) when MV is considered, RID policy is unable to reflect all costs since it has no way to infer the uninspected unit quality from the inspected unit quality. In fact, two kinds of penalty while performing RIDincorrect acceptance and rejection-are ignored in Raz et al.'s [6] cost model. (ii) Although Raz et al. [6] present several discussions of a process with a non-constant failure rate, their presentation form is difficult to use due to the lack of an explicit cost function in which many conditional probabilities are contained. In addition, a linear search is necessary to compute the BEP which results in increased computational effort.

Therefore, in this paper, we reformulated Raz et al.'s [6] model to consider MV and a case where the process has a non-constant failure rate. This included extra penalty costs and an additional index $f$ to record the original position of the discussed batch.

An explicit cost function for our developed ID model is given. A closed form of the break-even point is also obtained to facilitate computing the cost of no inspection when the process has a discrete Weibull shift distribution. This will facilitate wider and easier adoption of the off-line ID model. The related literatures of quality control in this paper are summarized in Table 1.

The remainder of this paper is organized as follows. In Section 2, we describe the ID model where the process possesses MV and NCFR. Later, an algorithm is proposed for the optimal ID policy. In Section 3, numerical examples are performed to illustrate the effects of MV and/or NCFR on the optimal ID policy. Concluding remarks are made in Section 4. 
Table 1: The related literature on quality control in this paper

Is there a product inspection policy introduced?

Yes No

Are non-conforming products repairable?

Yes No

Wang and Sheu[8] He et al.[3], Raz et al.[6] and this study Porteus[5]

\section{The Model and Algorithm}

Considering a batch is produced from the following description of an unreliable process. The process state is classified as either in-control or out-of-control. When the process is in-control (out-of-control), there is a percentage of $\theta_{1}\left(\theta_{2}\right)$ and $1-\theta_{1}\left(1-\theta_{2}\right)$ conforming items and non-conforming items produced, respectively. Note that $1 \geq \theta_{1}>\theta_{2} \geq 0$ is assumed. At the beginning of each production run, the process is setup and maintained to be in-control. The number of the items produced items since the last setup before the process shifts from the in-control state to the out-of-control state is a random variable $Y$. Let $\operatorname{Pr}(Y>j)=\bar{P}_{j}$ represent the probability that the first $j$ units are produced in the in-control state. This would not lose generality when a discrete Weibull shift distribution is used to model the process reliability. That is, $\bar{P}_{j}=p^{j^{\alpha}}$, where $0<p<1$ and $\alpha>0$. The property of a decreasing, constant and increasing process failure rate can be characterized as $0<\alpha<1, \alpha=1$ and $\alpha>1$, respectively (see Nakagawa and Osaki [4]).

Our objective here is to determine the optimal ID policy for a batch of size $k$, starting from unit $f$, which is produced from the above described process. The corresponding cost is given by

$$
V(f, k)=\min \left\{\min _{1 \leq j \leq k} V^{1}(f, k ; j), V^{0}(f, k)\right\}
$$

where $V^{1}(f, k ; j)$ and $V^{0}(f, k)$ represent the cost of a batch of size $k$, starting from unit $f$ when the $j$ th unit in this batch is inspected and not a unit in this batch is inspected, respectively.

Note that based on Raz et al.'s [6] ID policy, once a non-conforming unit is found, all subsequently produced units are rejected and the rest of the units including the found nonconforming unit are designated as the remaining batch for further ID decision. Thus, we will encounter the case where the last unit in the batch is non-conforming. This requires that we determine the optimal ID policy for a batch of size $k$, starting from unit $f$, where the last unit in this batch has been inspected to be non-conforming, with optimal cost denoted by $G(f, k)$. More precisely, we have

$$
G(f, k)=\min \left\{\min _{1 \leq j \leq k-1} G^{1}(f, k ; j), G^{0}(f, k)\right\}
$$

where $G^{1}(f, k ; j)$ and $G^{0}(f, k)$ represent the cost of a batch of size $k$, starting from unit $f$, and the last unit in this batch has been inspected to be non-conforming when the $j$ th unit in this batch is inspected and not a unit in this batch is inspected, respectively. 
In the following context, we first comply with the no inspection policy and then proceed to include the decision to perform inspection for the optimal ID policy. A batch of size $k$, starting from unit $f$ is considered if there is no particular specification.

\subsection{No inspection policy}

Let indicator variable $X_{i}=0$ or 1 represent whether the unit $i$ is inspected to be nonconforming or conforming, respectively. We first consider the case in which there is no inspection information in the batch. Conditioning on the random variable $Y$, the probability that the $j$ th unit in the batch (i.e., unit $f+j-1$ ) will be produced as a non-conforming unit is given by

$$
\operatorname{Pr}\left(X_{f+j-1}=0\right)=1-\theta_{2}+\left(\theta_{2}-\theta_{1}\right) \bar{P}_{f+j-1} .
$$

The probability that the $j$ th unit in the batch will be produced as a conforming unit can be computed as $1-\operatorname{Pr}\left(X_{f+j-1}=0\right)$, i.e.,

$$
\operatorname{Pr}\left(X_{f+j-1}=1\right)=\left(\theta_{1}-\theta_{2}\right) \bar{P}_{f+j-1}+\theta_{2} .
$$

When no inspection is considered, we must find the break-even point (BEP) for which the penalty for incorrect acceptance and incorrect rejection are equal. It is then economical to accept (reject) those units that are produced prior to (after) the BEP. This is because when the original position of the produced unit increases, the non-conforming probability increases, as can be seen from Equation (1), and hence the incorrect acceptance penalty increases in the original position. The BEP can be obtained by solving the following equation

$$
C_{p} \operatorname{Pr}\left(X_{f+j-1}=0\right)=C_{s} \operatorname{Pr}\left(X_{f+j-1}=1\right),
$$

where $C_{p}\left(C_{s}\right)$ is the cost penalty for accepting (rejecting) a non-conforming (conforming) unit. The last equation can be written as

$$
C_{p}\left(\left(\theta_{2}-\theta_{1}\right) \bar{P}_{f+j-1}+1-\theta_{2}\right)=C_{s}\left(\left(\theta_{1}-\theta_{2}\right) \bar{P}_{f+j-1}+\theta_{2}\right) .
$$

Substituting $\bar{P}_{j}=p^{j^{\alpha}}$ into the last equation and rearranging it gives:

$$
j^{*}=\min \left\{\max \left\{\left\lfloor\left\{\log \left(\left(C_{p} /\left(C_{p}+C_{s}\right)-\theta_{2}\right) /\left(\theta_{1}-\theta_{2}\right)\right) / \log (p)\right\}^{1 / \alpha}\right\rfloor-f+1,0\right\}, k\right\} .
$$

The last equation implies that all items in the batch will be accepted if the $\operatorname{BEP}\left(j^{*}\right)$ is greater or equal to $k$, and all items will be rejected if the BEP is less or equal to zero. Moreover, if we set $\alpha=1, f=1, \theta_{1}=1$ and $\theta_{2}=0$ in the last equation, it is easy to verify that the BEP is the same as given in Raz el al. [6]:

$$
j^{*}=\min \left\{\max \left\{\left\lfloor\log \left(C_{p} /\left(C_{p}+C_{s}\right)\right) / \log (p)\right\rfloor, 0\right\}, k\right\} .
$$

The expected total cost of the no inspection policy contains the penalty incurred by the acceptance of non-conforming units between $f$ and $f+j^{*}-1$, and the penalty incurred by the rejection of conforming units between $f+j^{*}$ and $f+k-1$. That is,

$$
\begin{aligned}
V^{0}(f, k) & =C_{p} \sum_{j=1}^{j^{*}} \operatorname{Pr}\left(X_{f+j-1}=0\right)+C_{s} \sum_{j=j^{*}+1}^{k} \operatorname{Pr}\left(X_{f+j-1}=1\right) \\
& =C_{p} \sum_{j=1}^{j^{*}}\left(\left(\theta_{2}-\theta_{1}\right) \bar{P}_{f+j-1}+1-\theta_{2}\right)+C_{s} \sum_{j=j^{*}+1}^{k}\left(\left(\theta_{1}-\theta_{2}\right) \bar{P}_{f+j-1}+\theta_{2}\right) .
\end{aligned}
$$


Once the last unit in the batch has been inspected to be non-conforming, the $j$ th unit in the batch will be produced as non-conforming with probability

$$
\begin{aligned}
& \operatorname{Pr}\left(X_{f+j-1}=0 \mid X_{f+k-1}=0\right) \\
& =\frac{\left(1-\theta_{2}\right)^{2}\left(1-\bar{P}_{f+j-1}\right)+\left(1-\theta_{1}\right)\left(1-\theta_{2}\right)\left(\bar{P}_{f+j-1}-\bar{P}_{f+k-1}\right)+\left(1-\theta_{1}\right)^{2} \bar{P}_{f+k-1}}{\left(1-\theta_{2}\right)\left(1-\bar{P}_{f+k-1}\right)+\left(1-\theta_{1}\right) \bar{P}_{f+k-1}}, 1 \leq j \leq k-1 .
\end{aligned}
$$

On the other hand, the $j$ th unit in the batch will be produced as conforming with probability

$$
\begin{aligned}
& \operatorname{Pr}\left(X_{f+j-1}=1 \mid X_{f+k-1}=0\right) \\
& =\frac{\theta_{2}\left(1-\theta_{2}\right)\left(1-\bar{P}_{f+j-1}\right)+\theta_{1}\left(1-\theta_{2}\right)\left(\bar{P}_{f+j-1}-\bar{P}_{f+k-1}\right)+\theta_{1}\left(1-\theta_{1}\right) \bar{P}_{f+k-1}}{\left(1-\theta_{2}\right)\left(1-\bar{P}_{f+k-1}\right)+\left(1-\theta_{1}\right) \bar{P}_{f+k-1}}, 1 \leq j \leq k-1 .
\end{aligned}
$$

The break even point can be easily obtained by solving the following equation:

$$
C_{p} \operatorname{Pr}\left(X_{f+j-1}=0 \mid X_{f+k-1}=0\right)=C_{s} \operatorname{Pr}\left(X_{f+j-1}=1 \mid X_{f+k-1}=0\right)
$$

Incorporating the conditional probabilities of the relevant events and substituting $\bar{P}_{j}=p^{j^{\alpha}}$ into the last equation gives:

$j^{\prime}=\min \left\{\max \left\{\left\lfloor\left\{\frac{\log \left(\frac{C_{p}\left(1-\theta_{2}\right)-\theta_{2} C_{s}}{\left(\theta_{1}-\theta_{2}\right)\left(C_{p}+C_{s}\right)}-\left(\frac{C_{p}\left(1-\theta_{1}\right)-\theta_{1} C_{s}}{\left(1-\theta_{2}\right)\left(C_{p}+C_{s}\right)}\right) p^{(f+k-1)^{\alpha}}\right)}{\log (p)}\right\}^{1 / \alpha}\right\rfloor-f+1,0\right\}, k-1\right\}$.

Set $\alpha=1, f=1, \theta_{1}=1$ and $\theta_{2}=0$ in the last equation and we have

$$
j^{\prime}=\min \left\{\max \left\{\left\lfloor\log \left(\left(C_{p}+C_{s} p^{k}\right) /\left(C_{p}+C_{s}\right)\right) / \log (p)\right\rfloor, 0\right\}, k-1\right\},
$$

which is exactly the result of Raz et al. [6]. Similar to the method used to obtain $V^{0}(f, k)$, the expected total cost of the no inspection policy when the last unit is non-conforming for a batch of size $k$, starting from unit $f$, is given by

$$
\begin{aligned}
& G^{0}(f, k) \\
& \quad=C_{p} \sum_{j=1}^{j^{\prime}} \operatorname{Pr}\left(X_{f+j-1}=0 \mid X_{f+k-1}=0\right)+C_{s} \sum_{j=j^{\prime}+1}^{k-1} \operatorname{Pr}\left(X_{f+j-1}=1 \mid X_{f+k-1}=0\right) \\
& \quad=C_{p} \sum_{j=1}^{j^{\prime}} \frac{\left(1-\theta_{2}\right)^{2}\left(1-\bar{P}_{f+j-1}\right)+\left(1-\theta_{1}\right)\left(1-\theta_{2}\right)\left(\bar{P}_{f+j-1}-\bar{P}_{f+k-1}\right)+\left(1-\theta_{1}\right)^{2} \bar{P}_{f+k-1}}{\left(1-\theta_{2}\right)\left(1-\bar{P}_{f+k-1}\right)+\left(1-\theta_{1}\right) \bar{P}_{f+k-1}} \\
& \quad+C_{s} \sum_{j=j^{\prime}+1}^{k-1} \frac{\theta_{2}\left(1-\theta_{2}\right)\left(1-\bar{P}_{f+j-1}\right)+\theta_{1}\left(1-\theta_{2}\right)\left(\bar{P}_{f+j-1}-\bar{P}_{f+k-1}\right)+\theta_{1}\left(1-\theta_{1}\right) \bar{P}_{f+k-1}}{\left(1-\theta_{2}\right)\left(1-\bar{P}_{f+k-1}\right)+\left(1-\theta_{1}\right) \bar{P}_{f+k-1}} .
\end{aligned}
$$

\subsection{The inspection policy}

Suppose there is no inspection information in the batch. When the $j$ th unit in the batch is inspected, which costs $C_{I}$, the resulting cost is analyzed as follows.

(1.) If the $j$ th unit in this batch is inspected to be non-conforming, which has probability $\operatorname{Pr}\left(X_{f+j-1}=0\right)$, then we reject units $f+j$ through $f+k-1$, and units $f$ through $f+j-1$ remain for further ID decision. Note that the total cost for the remaining batch is $G(f, j)$ since the last unit in the batch (i.e., unit $f+j-1$ ) is non-conforming. 
(2.) By contrast, if the $j$ th unit in the batch is inspected to be conforming, which has probability $\operatorname{Pr}\left(X_{f+j-1}=1\right)$, we accept units $f$ through $f+j-1$, and units $f+j$ through $f+k-1$ remain for further ID decision. The cost for the remaining batch is $V(f+j, k-j)$.

The expected total cost when the $j$ th unit in the batch is inspected is given by

$$
\begin{aligned}
V^{1}( & f, k ; j) \\
& =C_{I}+\operatorname{Pr}\left(X_{f+j-1}=0\right)\left[G(f, j)+C_{s} \sum_{i=f+j}^{f+k-1} \operatorname{Pr}\left(X_{i}=1 \mid X_{f+j-1}=0\right)\right] \\
& +\operatorname{Pr}\left(X_{f+j-1}=1\right)\left[V(f+j, k-j)+C_{p} \sum_{i=f}^{f+j-2} \operatorname{Pr}\left(X_{i}=0 \mid X_{f+j-1}=1\right)\right] \\
& =C_{I}+\left(\left(\theta_{2}-\theta_{1}\right) \bar{P}_{f+j-1}+\left(1-\theta_{2}\right)\right) G(f, j) \\
& +C_{s} \sum_{i=f+j}^{f+k-1}\left[\theta_{2}\left(1-\theta_{2}\right)\left(1-\bar{P}_{f+j-1}\right)+\theta_{2}\left(1-\theta_{1}\right)\left(\bar{P}_{f+j-1}-\bar{P}_{i}\right)+\theta_{1}\left(1-\theta_{1}\right) \bar{P}_{i}\right] \\
& +\left(\left(\theta_{1}-\theta_{2}\right) \bar{P}_{f+j-1}+\theta_{2}\right) V(f+j, k-j) \\
& +C_{p} \sum_{i=f}^{f+j-2}\left[\left(1-\theta_{2}\right) \theta_{2}\left(1-\bar{P}_{i}\right)+\left(1-\theta_{1}\right) \theta_{2}\left(\bar{P}_{i}-\bar{P}_{f+j-1}\right)+\left(1-\theta_{1}\right) \theta_{1} \bar{P}_{f+j-1}\right] .
\end{aligned}
$$

Now we consider the case in which the last unit of the batch has been inspected to be non-conforming. If

(1.) the $j$ th unit in the batch is being inspected to be non-conforming, we reject units $f+j$ through $f+k-1$, and units $f$ through $f+j-1$ remain. Note that the cost for the remaining batch is $G(f, j)$ since the last item in the batch (i.e., item $f+j-1$ ) is still non-conforming.

(2.) On the other hand, if the $j$ th unit is being inspected to be conforming, we accept units $f$ through $f+j-1$, and units $f+j$ through $f+k-1$ remain. Also note that the cost for the remaining batch is $G(f+j, k-j)$ since the last item in the batch (i.e., unit $f+k-1)$ is non-conforming.

Consequently, the expected total cost when the $j$ th unit in the batch is inspected, given that the last unit has been inspected to be non-conforming, is given by

$$
\begin{aligned}
& G^{1}(f, k ; j) \\
& \quad=C_{I}+\operatorname{Pr}\left(X_{f+j-1}=0\right)\left[G(f, j)+C_{s} \sum_{i=f+j}^{f+k-1} \operatorname{Pr}\left(X_{i}=1 \mid X_{f+j-1}=0\right)\right] \\
& \quad+\operatorname{Pr}\left(X_{f+j-1}=1\right)\left[V(f+j, k-j)+C_{p} \sum_{i=f}^{f+j-2} \operatorname{Pr}\left(X_{i}=0 \mid X_{f+j-1}=1\right)\right] \\
& \quad=C_{I}+ \\
& \quad\left(\frac{\left(1-\theta_{2}\right)^{2}\left(1-\bar{P}_{f+j-1}\right)+\left(1-\theta_{1}\right)\left(1-\theta_{2}\right)\left(\bar{P}_{f+j-1}-\bar{P}_{f+k-1}\right)+\left(1-\theta_{1}\right)^{2} \bar{P}_{f+k-1}}{\left(1-\theta_{2}\right)\left(1-\bar{P}_{f+k-1}\right)+\left(1-\theta_{1}\right) \bar{P}_{f+k-1}}\right) \\
& \quad \times G(f, j)+\left(\frac{C_{s}}{\left(\theta_{2}-\theta_{1}\right) \bar{P}_{f+k-1}+\left(1-\theta_{2}\right)}\right)
\end{aligned}
$$




$$
\begin{aligned}
& \times \sum_{i=f+j}^{f+k-2}\left[\left(1-\theta_{2}\right)^{2} \theta_{2}\left(1-\bar{P}_{f+j-1}\right)+\left(1-\theta_{1}\right) \theta_{2}\left(1-\theta_{2}\right)\left(\bar{P}_{f+j-1}-\bar{P}_{i}\right)\right. \\
& \left.+\left(1-\theta_{1}\right) \theta_{1}\left(1-\theta_{2}\right)\left(\bar{P}_{i}-\bar{P}_{f+k-1}\right)+\left(1-\theta_{1}\right)^{2} \theta_{1} \bar{P}_{f+k-1}\right] \\
& +\left(\frac{\theta_{2}\left(1-\theta_{2}\right)\left(1-\bar{P}_{f+j-1}\right)+\theta_{1}\left(1-\theta_{2}\right)\left(\bar{P}_{f+j-1}-\bar{P}_{f+k-1}\right)+\theta_{1}\left(1-\theta_{1}\right) \bar{P}_{f+k-1}}{\left(1-\theta_{2}\right)\left(1-\bar{P}_{f+k-1}\right)+\left(1-\theta_{1}\right) \bar{P}_{f+k-1}}\right) \\
& \times G(f+j, k-j)+\left(\frac{C_{p}}{\left(\theta_{2}-\theta_{1}\right) \bar{P}_{f+k-1}+\left(1-\theta_{2}\right)}\right) \\
& \times \sum_{i=f}^{f+j-2}\left[\left(1-\theta_{2}\right)^{2} \theta_{2}\left(1-\bar{P}_{i}\right)+\left(1-\theta_{1}\right) \theta_{2}\left(1-\theta_{2}\right)\left(\bar{P}_{i}-\bar{P}_{f+j-1}\right)\right. \\
& \left.+\left(1-\theta_{1}\right) \theta_{1}\left(1-\theta_{2}\right)\left(\bar{P}_{f+j-1}-\bar{P}_{f+k-1}\right)+\left(1-\theta_{1}\right)^{2} \theta_{1} \bar{P}_{f+k-1}\right] .
\end{aligned}
$$

To understand the work loads of the inspection facility, the expected number of inspections for a batch should be calculated. Let $I_{V}(f, k)$ and $I_{G}(f, k)$ represent the expected number of inspections for a batch of size $k$, starting from unit $f$, given that the quality information of the last unit in this batch is unknown and non-conforming, respectively. Then, $I_{V}(f, k)$ and $I_{G}(f, k)$ can be obtained through the following two recursive formulae:

$$
\begin{gathered}
I_{V}(f, k)=\left\{\begin{array}{l}
0, \text { if } V(f, k)=V^{0}(f, k) \\
1+\operatorname{Pr}\left(X_{f+v^{*}(f, k)-1}=0\right) I_{G}\left(f, v^{*}(f, k)\right) \\
+\operatorname{Pr}\left(X_{f+v^{*}(f, k)-1}=1\right) I_{V}\left(f+v^{*}(f, k), k-v^{*}(f, k)\right), \text { otherwise }
\end{array}\right. \\
I_{G}(f, k)=\left\{\begin{array}{l}
0, \text { if } G(f, k)=G^{0}(f, k), \\
1+\operatorname{Pr}\left(X_{f+g^{*}(f, k)-1}=0 \mid X_{f+k-1}=0\right) I_{G}\left(f, g^{*}(f, k)\right) \\
+\operatorname{Pr}\left(X_{f+g^{*}(f, k)-1}=1 \mid X_{f+k-1}=0\right) \\
\times I_{G}\left(f+g^{*}(f, k), k-g^{*}(f, k)\right), \text { otherwise }
\end{array}\right.
\end{gathered}
$$

where $g^{*}(f, k)=\arg \min _{1 \leq j \leq k-1} G^{1}(f, k ; j)$ and $v^{*}(f, k)=\arg \min _{1 \leq j \leq k} V^{1}(f, k ; j)$.

\subsection{Algorithm for optimal ID policy}

Based on the above analysis, the following algorithm is proposed to compute the minimum cost and the expected number of inspections for the optimal ID policy, where a batch of size $N$, starting from unit one is considered.

Step 1. Input the value for these parameters: $\theta_{1}, \theta_{2}, N, C_{I}, C_{p}, C_{s}$ and $\bar{P}_{j}$, for $j=$ $1,2, \cdots, N$.

Step 2. For $k=1,2, \cdots, N-f+1$, compute the break-even points $j^{*}$ and $j^{\prime}$ at $f=$ $1,2, \cdots, N$ and $f=1,2, \cdots, N-1$, respectively.

Step 3. Using $j^{*}$ and $j^{\prime}$ obtained in Step 2, determine the costs of no inspection $V^{0}(f, k)$ and $G^{0}(f, k)$ using Equation (2) and Equation (3), respectively.

Step 4. Set the initial conditions: $V(f, 0)=0, G(f, 1)=0, I_{V}(f, 0)=0$ and $I_{G}(f, 1)=0$, $f=1,2, \cdots, N$.

Step 5 . With increasing values of $k=2,3, \cdots, N$, sequentially compute

$G(f, k)=\min \left\{\min _{1 \leq j \leq k-1} G^{1}(f, k ; j), G^{0}(f, k)\right\}$ and $I_{G}(f, k)$ using Equation $(7)$, for $f=$ $1,2, \cdots$,

$N-k+1$, where $G^{1}(f, k ; j)$ can be obtained from Equation (5). 
Step 6. With increasing values of $k=1,2, \cdots, N$, sequentially compute

$V(f, k)=\min \left\{\min _{1 \leq j \leq k} V^{1}(f, k ; j), V^{0}(f, k)\right\}$ and $I_{V}(f, k)$ using Equation $(6)$, for $f=$ $1,2, \cdots$,

$N-k+1$, where $V^{1}(f, k ; j)$ can be obtained from Equation (4).

Step 7. Output $V(1, N)$ and $I_{V}(1, N)$ are the minimum cost and the expected number of inspections, respectively, for the optimal ID policy. STOP.

\section{Numerical Examples}

In this section, we present a numerical example to illustrate the effects of $\mathrm{MV}$ and/or NCFR on the optimal ID policy. The discrete Weibull shift distribution is used for the analysis of the numerical example, i.e., $\bar{P}_{j}=p^{j^{\alpha}}$. As in Raz et al. [6], the unit cost for inspection, incorrect acceptance and incorrect rejection, $C_{I}, C_{p}$ and $C_{s}$, are selected with ten combinations (namely cases $\mathrm{A}$ to $\mathrm{J}$ ) that suffice for the policy of perfect information (i.e., case A), zero-defects (i.e., case B) and cost minimization (i.e., cases $\mathrm{C}$ to J), where cases C-E, cases F-G, and cases H-J are performed for the sensitivity analysis of parameters $C_{p}$, $C_{s}$ and $C_{I}$, respectively. For each of the ten cases, various combinations of the parameter values $\alpha, \theta_{1}$ and $\theta_{2}$ are selected to investigate the changes in $I_{V}(1, N)$ and $V(1, N)$, where $\left(\alpha, \theta_{1}, \theta_{2}\right)$ take on the values of $(1,1,0),(1,0.99,0.01),(1.3,1,0)$ and $(1.3,0.99,0.01)$. The results are shown in Tables 2-5, respectively, where FIU means the first inspected unit in this batch, the asterisk represents that inspection is never economically justified for these combinations, $10^{6}$ is used for the infinite value and cost error (CE) is defined as Raz et al.'s result minus our result. Note that Table 2 shows the results for Raz et al.'s [6] model and Tables 3-5 are the results for our model.

Comparison among Tables 2-5 revealed that

(i) When MV does not exist (see Tables 2 and 4), inspection gives exact information about the process status as it produces the unit being inspected. Furthermore, when the process has a higher failure rate (i.e., $\alpha=1.3$ ), it takes a lower number of inspections and has a smaller minimum cost (see Table 4).

(ii) For the same process failure rate, (i.e., a fixed $\alpha$ ), the number of inspections and minimum cost are larger when MV exists (see Tables 2-5).

(iii) When MV exists (see Tables 3 and 5), the number of inspections and minimum cost do not exhibit monotone behavior for different failure rates (i.e., $\alpha=1$ and $\alpha=1.3$ ). In addition, it is infeasible to reach the perfect information policy once MV exists, but the zero-defects policy is still feasible. Furthermore, when the process has an IFR (see Table 5 ), it is shown that as the inspection cost is is relatively high in comparison to $C_{p}$ and $C_{s}$, it would require no inspection (see $\mathrm{H}$ and $\mathrm{I}$ ). From most of the cases given in Table 5 , we found that the cost error was significant. As a result, It would be better to avoid using Raz et al.'s [6] model when both MV and IFR exist.

(vi) For each combination of cases $\mathrm{A}$ to $\mathrm{J}$, we use the numerical results given in Tables 3-5 to compute their root mean squared error (RMSE) on results from using Raz et al.'s [6] model instead of ours, when MV and/or NCFR does exist. The term RMSE is computed by $\left\{\sum_{j=3}^{5}\left(\mathrm{CE}^{2} \text { of Table } j\right) / 3\right\}^{1 / 2}$. For example, the RMSE for case $\mathrm{E}$ can be obtained by $\left\{\left[(-4.92)^{2}+(1.12)^{2}+(-8.45)^{2}\right] / 3\right\}^{1 / 2}=5.68$. The results are given in Table 6. Moreover, Table 6 reveals that the perfect information (i.e., case A) cannot be 
Table 2: The optimal ID policy, neither MV nor NCFR are considered

\begin{tabular}{lrrrrrrr} 
& & & \multicolumn{5}{c}{$p=0.99, \alpha=1, \theta_{1}=1, \theta_{2}=0$} \\
\cline { 6 - 8 } & $C_{I}$ & $C_{p}$ & $C_{s}$ & $\mathrm{FIU}$ & $I_{V}(1, N)$ & $V(1, N)$ & $\mathrm{CE}$ \\
\hline $\mathrm{A}$ & 1 & $10^{6}$ & $10^{6}$ & 1 & 5.19 & 5.19 & 0 \\
$\mathrm{~B}$ & 1 & $10^{6}$ & 1 & 2 & 4.17 & 4.81 & 0 \\
$\mathrm{C}$ & 1 & 50 & 10 & 2 & 5.19 & 5.19 & 0 \\
$\mathrm{D}$ & 1 & 10 & 10 & 5 & 5.19 & 5.19 & 0 \\
$\mathrm{E}$ & 1 & 1 & 10 & 18 & 3.98 & 4.72 & 0 \\
$\mathrm{~F}$ & 1 & 10 & 50 & 5 & 5.19 & 5.19 & 0 \\
$\mathrm{G}$ & 1 & 10 & 1 & 5 & 4.17 & 4.81 & 0 \\
$\mathrm{H}$ & 50 & 1 & 1 & $*$ & 0 & 32.73 & 0 \\
$\mathrm{I}$ & 10 & 1 & 1 & 59 & 1 & 20.59 & 0 \\
$\mathrm{~J}$ & 1 & 1 & 1 & 17 & 3.45 & 4.38 & 0 \\
\hline
\end{tabular}

Table 3: The optimal ID policy when MV is considered

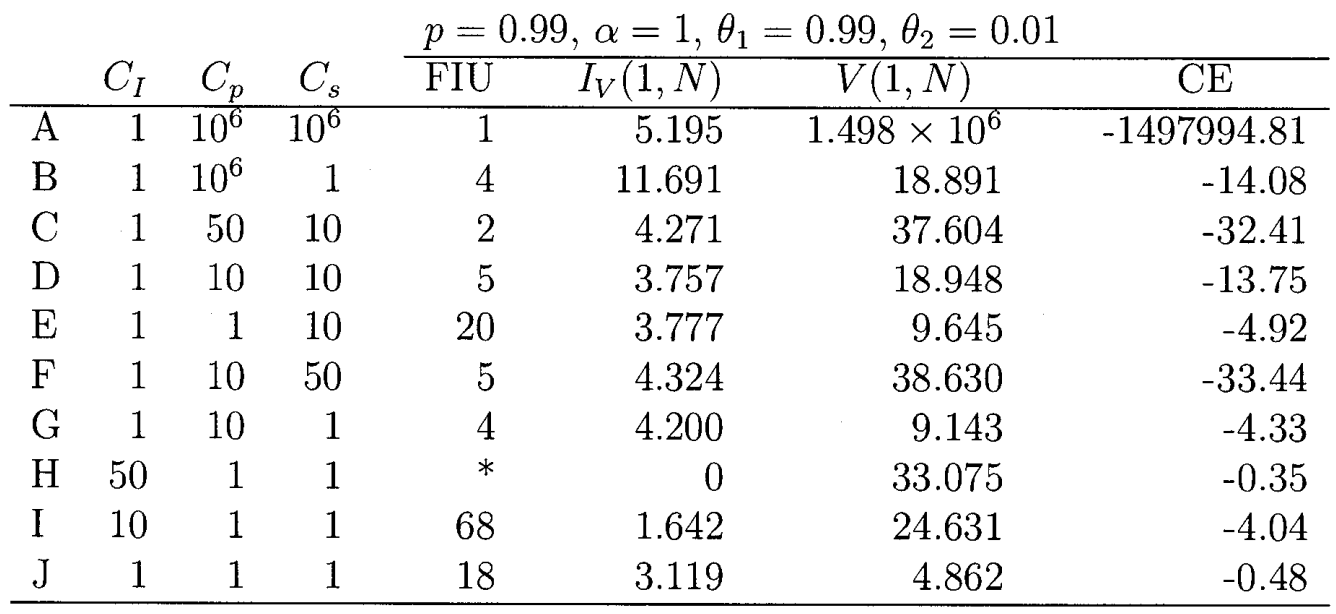

obtained at a reasonable cost, as Raz et al. [6] claim. For cost minimization policy (i.e., cases $\mathrm{C}$ to $\mathrm{J}$ ), the RMSE is significant when one of the three costs $C_{p}, C_{s}$ or $C_{I}$ is large (see cases $\mathrm{C}, \mathrm{F}$ and $\mathrm{H}$ ).

(v) Finally, the FIU did not exhibit a particular behavior in the results of Tables 2-5.

\section{Conclusion}

This study has provided a solution to the economic optimization of an offline inspection/disposition (ID) model where the process deterioration follows a discrete Weibull shift distribution with a non-constant failure rate (NCFR) and the existence of manufacturing variation (MV) is considered. The incorporation of the two features, MV and NCFR, into the ID model extend its applicability. Once MV exists, the inspection information is not sufficient to determine the process's actual status. Therefore, two kinds of extra penalty while performing RID --incorrect acceptance and rejection - are included in our model. Numerical examples show that it would be better to avoid using Raz et al.'s [6] model when both MV and IFR exist. Also, when MV exists the perfect information policy is infeasible 
Table 4: The optimal ID policy when NCFR is considered

\begin{tabular}{|c|c|c|c|c|c|c|c|}
\hline & \multirow[b]{2}{*}{$C_{I}$} & \multirow[b]{2}{*}{$C_{p}$} & \multirow[b]{2}{*}{$C_{s}$} & \multicolumn{4}{|c|}{$p=0.99, \alpha=1.3, \theta_{1}=1, \theta_{2}=0$} \\
\hline & & & & FIU & $I_{V}(1, N)$ & $V(1, N)$ & $\mathrm{CE}$ \\
\hline $\mathrm{A}$ & 1 & $10^{6}$ & $10^{6}$ & 1 & 3.646 & 3.646 & 1.55 \\
\hline $\mathrm{B}$ & 1 & $10^{6}$ & 1 & 2 & 3.123 & 3.420 & 1.39 \\
\hline $\mathrm{C}$ & 1 & 50 & 10 & 2 & 3.645 & 3.646 & 1.55 \\
\hline $\mathrm{D}$ & 1 & 10 & 10 & 4 & 3.645 & 3.646 & 1.55 \\
\hline $\mathrm{E}$ & 1 & 1 & 10 & 14 & 3.648 & 3.604 & 1.12 \\
\hline F & 1 & 10 & 50 & 4 & 3.646 & 3.646 & 1.55 \\
\hline $\mathrm{G}$ & 1 & 10 & 1 & 4 & 3.123 & 3.420 & 1.39 \\
\hline $\mathrm{H}$ & 50 & 1 & 1 & $*$ & 0 & 18.200 & 14.52 \\
\hline I & 10 & 1 & 1 & 88 & 1 & 18.014 & 2.58 \\
\hline $\mathrm{J}$ & 1 & 1 & 1 & 14 & 3.025 & 3.374 & 1.01 \\
\hline
\end{tabular}

Table 5: The optimal ID policy when both MV and NCFR are considered

\begin{tabular}{lrrrrrrr} 
& & & \multicolumn{6}{c}{$p=0.99, \alpha=1.3, \theta_{1}=0.99, \theta_{2}=0.01$} \\
\cline { 6 - 8 } & $C_{I}$ & $C_{p}$ & $C_{s}$ & FIU & $I_{V}(1, N)$ & $V(1, N)$ & \multicolumn{1}{c}{ CE } \\
\hline $\mathrm{A}$ & 1 & $10^{6}$ & $10^{6}$ & 1 & 4.774 & $1.254 \times 10^{6}$ & -1253994.81 \\
$\mathrm{~B}$ & 1 & $10^{6}$ & 1 & 4 & 8.983 & 12.128 & -7.31 \\
$\mathrm{C}$ & 1 & 50 & 10 & 2 & 3.900 & 24.101 & -18.91 \\
$\mathrm{D}$ & 1 & 10 & 10 & 4 & 3.707 & 16.384 & -11.19 \\
$\mathrm{E}$ & 1 & 1 & 10 & 16 & 4.018 & 13.170 & -8.45 \\
$\mathrm{~F}$ & 1 & 10 & 50 & 4 & 5.017 & 50.796 & -45.6 \\
$\mathrm{G}$ & 1 & 10 & 1 & 4 & 3.363 & 6.506 & -1.69 \\
$\mathrm{H}$ & 50 & 1 & 1 & $*$ & 0 & 18.836 & 13.89 \\
$\mathrm{I}$ & 10 & 1 & 1 & $*$ & 0 & 18.836 & 1.76 \\
$\mathrm{~J}$ & 1 & 1 & 1 & 14 & 2.993 & 4.697 & -0.31 \\
\hline
\end{tabular}

Table 6: RMSE for the existence of MV and/or NCFR

\begin{tabular}{rrrrrrrrrrrrr}
\hline & & & & B & C & D & E & F & G & H & I & J \\
\hline RMSE & 1127902.39 & 9.19 & 21.68 & 10.27 & 5.68 & 32.65 & 2.80 & 11.60 & 2.94 & 0.66 \\
\hline
\end{tabular}


but the zero-defects policy is still feasible. A possible extension of this study is to constrain the average outgoing quality level or supply contract of quality to determine the associated optimal ID policy.

Acknowledgments The authors wish to express their appreciation to the anonymous referees for their very valuable comments and suggestions, which greatly enhanced the clarity of the article. All their suggestions were incorporated directly into the text. This research was partially supported by the National Science Council of Taiwan under Grant NSC 942213-E-214-024-

\section{References}

[1] T. Brosnan and D.W. Sun: Inspection and grading of agricultural and food products by computer version system-a review. Computers and Electronic in Agriculture, 36 (2002), 193-213.

[2] I. Djamaludin, D.N.P. Murthy, and R.J. Wilson: Quality control through lot sizing for items sold with warranty. International Journal of Production Economics, 33 (1994), 97-107.

[3] Q.M. He, Y. Gerchak and A. Grossfeld-Nir: Optimal inspection order when process failure rate is constant. International Journal of Reliability, Quality and Safety Engineering, 3-1 (1996), 25-41.

[4] T. Nakagawa and S. Osaki: The discrete Weibull distribution. IEEE Transactions on Reliability, R-24-5 (1975), 300-301.

[5] E.L. Porteus: Optimal lot sizing, process quality improvement and setup cost reduction. Operations Research, 34 (1986), 137-144.

[6] T. Raz, Y.T. Herer, and A. Grosfeld-Nir: Economic optimization of off-line inspection. IIE Transactions, 32 (2000), 205-217.

[7] S.H. Sheu, Y.C. Chen, W.Y. Wang, and N.H. Shin: Economic off-line inspection with inspection errors. Journal of the Operational Research Society, 54 (2003), 888-895.

[8] C.H. Wang and S.H. Sheu: Simultaneous determination of the optimal productioninventory and product inspection policies for a deteriorating production system. Computers and Operations Research, 28 (2001), 1093-1110.

[9] C.H. Wang: Economic off-line quality control strategy with two types of inspection errors. European Journal of Operational Research, 179-1 (2007), 132-147.

[10] L. Wang, C. Chessari, and E. Karpiel: Inferential control of product quality attributesapplication to food cooking extrusion process. Journal of Process Control, 11 (2001), 621-636.

Chih-Hsiung Wang

National Pingtung Institute of Commerce

51 Min-Sheng E. Road, Pingtung 900, Taiwan

E-mail: chwang@npic.edu.tw 